\title{
The influence of social factors on the control of asthma
}

\author{
C.K. Connolly, N.S. Chan* and R.J. Prescott ${ }^{1}$
}

Darlington Memorial Hospital, Darlington, Co Durham and ${ }^{1}$ Medical Statistics Unit, Medical School, Teviot Place, Edinburgh, $U K$.

\begin{abstract}
Summary: The relationships of pulmonary function with age, duration of asthma and social factors were studied in $\mathbf{6 3 0}$ asthmatics. Pulmonary function was assessed by peak flow rate at routine attendance (actual function). Persistence of obstruction was demonstrated by a trial of corticosteroids where necessary. The best obtainable peak flow rate was recorded (maximum function). Unrelieved potentially reversible obstruction was assessed by actual/maximum function. Age and duration of asthma were directly associated with poor control of reversible wheeze as well as with persistent obstruction. In addition poor control of potentially reversible wheeze was related to lower social class, current cigarette smoking and lack of central heating. Persistent obstruction was related to life time amount smoked, passive smoking and lack of central heating.
\end{abstract}

\section{Introduction}

In the study of all 630 asthmatics attending one of the authors clinics, ${ }^{1}$ it was shown that persistent obstruction was related to age as well as duration of asthma. ${ }^{2}$ Further analysis was performed to establish the additional effect of social factors on the presence of persistent obstruction. At the same time, by comparing actual function at attendance with the best function demonstrable, the effect of age, duration of asthma, and social factors on control of the potentially reversible wheeze was assessed.

\section{Methods}

These have been described in detail previously. ${ }^{1-3}$

\section{Details of subjects}

All patients attending with the clinical diagnoses of asthma confirmed at some time by reversibility of peak flow rate (PEFR) or forced expiratory volume in one second $\left(\mathrm{FEV}_{1}\right)$ to bronchodilator or spontaneously on four hourly peak flow chart were included. The history included childhood asthma, household details (presence of central heating, children, animals and cigarette smokers), place of employment, employment status (males 25-59), and smoking history. Exsmokers were checked by carboxyhaemoglobin levels. Social class was determined by the principal male member of the household.

Correspondence: C.K. Connolly, M.A., M.B., F.R.C.P.

* Present address: Green Lane Hospital, Auckland 3, New Zealand.

Accepted: 28 November 1988

\section{Pulmonary function}

Actual PEFR Peak flow rate at attendance before bronchodilator was expressed as percentage predicted. ${ }^{4}$ The difference between this and 100\%@s measure of overall control at attendance.

Maximum PEFR The best recorded after bronêt dilator, within 12 months of attendance. If less tha $80 \%$ predicted four hourly recordings were require together with inhaled steroids or disodium cromogly cate (if $70 \%-80 \%$ predicted) or oral steroids ( $<70 \%$ predicted). ${ }^{\prime}$ The difference between the max mum PEFR and $100 \%$ is a measure of persister obstruction.

Actual/maximum PEFR This is expressed as percentage. The difference between this and $100 \%$ is measure of control of asthma at attendance allowing for any persistent obstruction.

Similar pulmonary variables derived from spirometery (actual $\mathrm{FEV}_{1}$, maximum forced vitat capacity (FVC), actual $\mathrm{FEV}_{1} / \mathrm{FVC}$ ) were calculated but the results are not presented in detail.

\section{Statistical methods}

For each of the respiratory variables in turn, the statistical significance of its association with each of the possible 'explanatory' variables was assessed using standard statistical methods. The explanator.y variables showing no significant associations with an of the respiratory variables were not considere

(C) The Fellowship of Postgraduate Medicine, 198 
further. Multiple regression was then used for each respiratory variable separately to establish which 'explanatory' variables still showed an independent significant association with pulmonary function. Analysis of covariance was applied subsequently to compare the regression equations in various subsets of the data, in order to investigate possible interactions between social variables in their effect on pulmonary function. All calculations were made using the BMDP statistical package. ${ }^{5}$

\section{Results}

Details of the 630 subjects were given previously. ${ }^{1}$ Three hundred and seventeen patients were observed on oral steroids and 224 patients on inhaled corticosteroids or sodium cromoglycate. ${ }^{3}$ Applying univariate analysis (full table available from the authors), atopy, pets and urban or rural residence had no significant effect on any respiratory variable, whether assessed by peak flow rate or spirometry, which on the whole gave weaker associations. Table I shows the regression coefficient and standard error of the 'explanatory' variables which were significant in the multivariate analysis for any of the peak flow variables. 'Children in the household' did not reach statistical significance at the $10 \%$ level in the multiple regression and therefore has not been included. Although 'ever smoked' is non-significant it is retained to allow a cleaner estimate of the effect of passive smoking. The previous paper ${ }^{1}$ shows that age is more important with respect to persistent obstruction in males than females so the age effect is shown separately for the sexes. Further regressions were calculated to check that there were no more interactions between the social variables. These included separate calculations for men and women, the different smoking categories, social class and age (above or below 55). The stratifications did not produce any further statistically significant differences in the regression coefficients.

The results show that age is relatively more important with control of potentially reversible wheeze and duration is more important with persistent obstruction. Log duration is shown because it fits to the data marginally better than actual duration. If the natural coefficients are used persistent obstruction increases at the rate of about $4 \%$ per decade of disease greater than the age effect in both sexes. Actual/maximum PEFR shows a reduction of only $1 \%$ per decade, which is less than the age effect. Lifetime amount smoked is important in its effect on persistent obstruction but not on the reversible component, while current smoking has a more pronounced effect on control of asthma. Passive smoking was associated with reduction in all three respiratory variables, though it did not remain significant for actual/maximum PEFR on multivariate analysis.

Poor control of asthma was strongly associated with lower social class. Although univariate analysis showed an association between class and persistent obstruction, this was no longer apparent on multivariate analysis, because of the very strong associa-

Table I Multiple regression analysis showing the factors significant for at least one pulmonary variable, including all the smoking variables

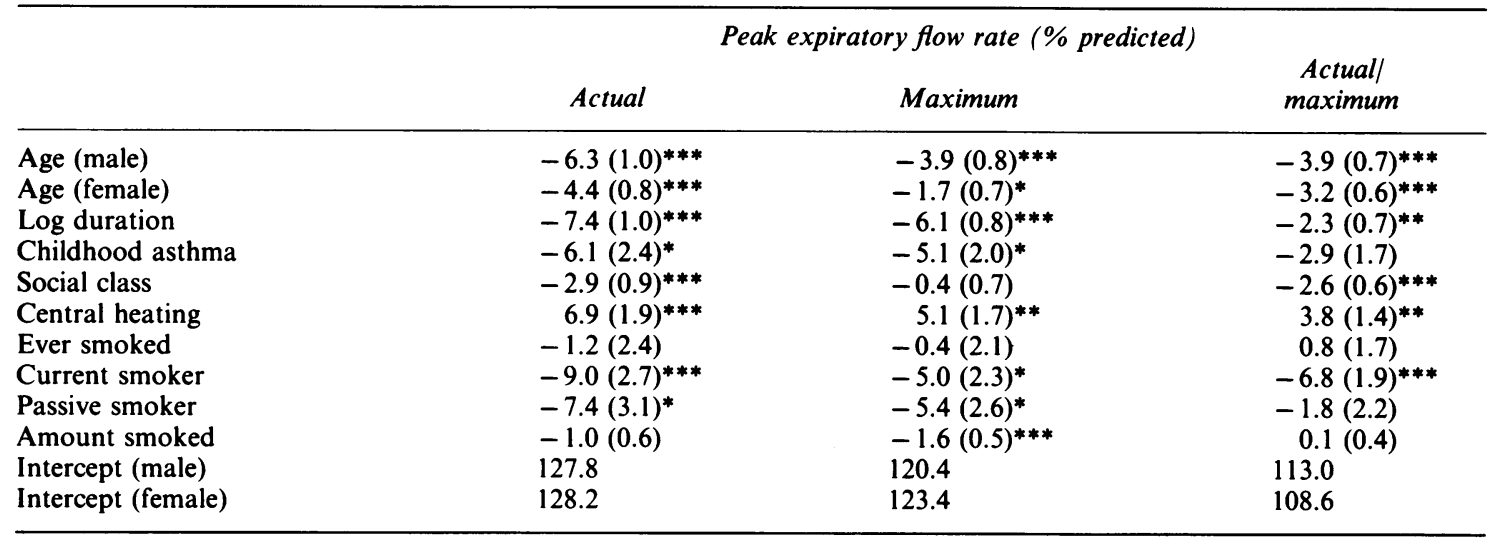

Significance: ${ }^{*} P<0.05,{ }^{* *} P<0.01,{ }^{* * *} P<0.001$.

The regression coefficients above are obtained from multiple regressions where: Age is in decades; log duration is log to base e of duration in years; childhood asthma $=0$ if absent, 1 if present; social class $=1$ to 5; central heating $=0$ if absent, 1 if present; smoking status is represented by 3 dummy variables each taking the value 1 if the condition is satisfied and 0 otherwise; amount smoked is expressed as pack years divided by 10 . 
tion between lack of central heating and persistent obstruction.

Assessment of employment was restricted to males between $25-59$ because it was difficult to interpret the significance of non-employment in females and males under 25 and over 60 . The employment rate of $79 \%$ was approximately that of the reference population. An increased chronic sickness rate $(12 \%$ in asthmatics $v s 6 \%$ in the general population) being balanced by decrease in unemployment rate $(6 \% v s 15 \%)$, with $2 \%$ of asthmatics prematurely retired. The sick and unemployed asthmatics had significantly more persistent obstruction than the retired and employed ( $\max$ PEFR corrected for age and duration $71 \%$ vs $86 \%$, $P<0.0002$ ), but the small difference in actual $/ \mathrm{max}$ PEFR was not significant. The effect of place of employment was inconsistent, though control was particularly good in places where there was an accepted hazard of respiratory disease.

\section{Discussion}

Pulmonary function at any one time in asthmatics depends on control of the potentially reversible obstruction. However, the patient's persistent obstruction and pulmonary function can never be better than the maximum achievable with optimal therapy. This study looks at both best or maximum function and control as assessed by actual/maximum function. To avoid the bias from unrepresentatively zealous compliance with treatment, it was necessary to give no warning of the study. Therefore repeated assessment of actual function was not practicable. Although the variability of asthma does reduce the value of single readings on single subjects, the numbers here do enable the mean of single readings to be taken as representative of the group as a whole. In a previous report from this study, ${ }^{3}$ it was shown that the relationship between persistent obstruction and poor control of potentially reversible wheeze was poor, accounting for less than $10 \%$ of the variance. This justifies looking at the two contributions to overall obstruction separately, and seeing how each is related to age, duration of asthma and social factors.
The relationships using peak flow were generalin much stronger than with the spirometric variables which gave similar associations between pulmonam function and the other parameters discussed here. These findings are in agreement with Mitchell et a $a t^{8}$ suggesting that PEFR is the more satisfactopy measurement in the assessment of asthma.

The previous report ${ }^{1}$ showed that duration $\frac{\vec{Q}}{\vec{A}}$ asthma was more closely associated with persiste obstruction than age, and this analysis suggests th poor control is more closely related to age tham duration. Even after age, duration and history of

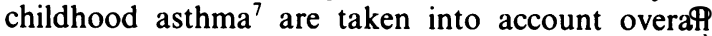
control of asthma is still strongly associated with various social factors. The persistent component related to lack of central heating but not to social cla after the former has been discounted. Occupation effects were small, but not only was the life timio number of cigarettes smoked an important determinant of persistent obstruction in smokers, but the was also a contribution from passive smoking iog non-smokers.

In contrast to persistent obstruction, poor contrg of the reversible component was associated with lower social class independently of lack of central heatin As might be expected the effect of current smoking wis much greater on reversible wheeze, than on the presence of persistent obstruction, once the strong effect of the total number of cigarettes smoked had been discounted.

Overall, the importance of central heating wit respect to both persistent obstruction and poor con trol suggests that standard of housing is of grea importance for asthmatics. The MRC cohort studye demonstrated this in childhood. Elderly asthmaticas have more wheeze than younger subjects. They res pond less well to optimal therapy, including cort costeroids, than younger subjects, but only a little more so than expected from the duration of asthma Less effective management of the reversible component is the principal reason for the age difference. The social class, housing (independently of this) and cigarette smoking appear to be highly relevant, bu work-related factors were not important determinants of poor pulmonary function in younger subjects. 


\section{References}

1. Connolly, C.K., Chan, N.S. \& Prescott, R.J. The relationship between age and duration of asthma and the presence of persistent obstruction in asthma. Postgrad Med J 1988, 64: $422-425$.

2. Brown, P.J., Greville, H.W. \& Finnicatine, K.E. Asthma and irreversible airflow obstruction. Thorax 1984, 39: $131-136$.

3. Connolly, C.K. \& Chan, N.S. The relationship between different measures of pulmonary function. Respiration 1987, 52: 22-32.

4. Cotes, J.E. Lung Function, 4 th edition. Blackwell Scientific Publications, Oxford, 1978.
5. Dixon, W.J., Brown, M.B., Engleman, L. et al. BMDP Statistical Software. University of California Press, Berkeley, Calif., 1983.

6. Mitchell, D.M., Gildeh, P., Rehahn, M., Dimond, A.H. \& Collins, J.V. Value of serial peak expiratory flow measurements in assessing treatment response in chronic airflow limitation. Thorax 1986, 41: 606-610.

7. Britten, N., Davies, J.M.C. \& Colley, J.R.I. Early respiratory experience and subsequent cough and peak flow rate in 36 year old men and women. Br Med J 1987, 294: $1317-1320$. 